\title{
Investigation on the Correlation of Interval Time Between Waking Up and Last REM, and Clarity of Dream Recalls
}

\begin{abstract}
Yichen $\mathrm{Li}^{1}$
Author Affiliation: United World College Changshu China

Corresponding Author Email: ycli20@uwcchina.org

ABSTRACT

When people wake up from a long night sleep, dreams are memorized, sometimes forgotten. What are the factors that influences the clarity and quality of dream recalls when people wake up? Data were collected from the author himself using EEG, survey and introspection. Totally 7 nights of data were collected for this investigation. The results demonstrated borderline negative correlation among the level of clarity of dream recall and the time interval between last REM and wake up. Results showed that the closer one woke up from REM sleep, the clearer the dream was recalled. Further improvement can be made on the variety of participant chosen and other possible factors that influence the clarity of dream recall.
\end{abstract}

Keywords: Dream, REM, Sleep, Dream Recall, Introspection

\section{INTRODUCTION}

When people wake up from a long night sleep, dreams are sometimes memorized, sometimes forgotten. Even when a dream can be recalled, there are different levels of clarity associated with the recall. This inspires the current study to investigate what are the factors that influences the clarity and quality of dream recalls at the time of wake-up. Since the discovery in 1953 of REM stage that composes half of adults' sleep with a repetition of seven to eight times and responsible for most dreams that take place at night [1], research has revealed that the ability of dream recall varies among individuals with some reporting dreams consistently and others failing to remember any content [2]. Time could also be a significant factor to impact the clarity of dream recall. A study showed that, $15 \%$ of the population reported decreased dream recall clarity 1 to 2 weeks after the dream [3]. Time interval between the time wakes up and closest REM is studied as influence factor on the clarity of dream recall after waking up.

\section{METHODOLOGY}

The investigation was conducted with an EGG headband as a case study. For this investigation, electrical waves from the brain were recorded to determine the time of REM. In addition, surveys were used to gather subjective data. Correlational analysis was performed on the data collected.

\subsection{Instrument Occupied}

For this investigation, Muse S Headband designed by the Company Muse ${ }^{\mathrm{TM}}$ was used to track brain activities during sleep. The headband, weight only $41 \mathrm{~g}$, contains built-in electrodes which are EGG sensors that can keeps track of brain activities and brain waves during sleep [4]. When the participant was sleeping, this headband was activated and record the brain waves, which were later processed offline. The processed data was presented as sleep stages at certain time intervals. These stages were categorized into "Awake", "Light Sleep", "Deep Sleep", and "REM". This data was used to determine the stage of sleep that the participant was currently in while being waked up. The timestamps of the onset and termination of each stage was also collected.

\subsection{Procedure}

Firstly, the participant activated the Muse App that comes along with the headband. After putting on Muse S headband properly, the participant checked for the connection and signal quality to make sure that the EGG sensor on the headband was functioning and collecting 
data. The complementary meditation course from the App was muted, and all lights were turned off while sleeping.

The next morning when the participant woke up, the time of waking up was recorded and the monitor session was closed. In order to create variety in the interval between sleep and REM, the time when the participant woke up were manipulated. To be more specific, alarm with random time was set every day. However, there were cases where the participant woke up before the alarm sounded. In these cases, the participant recorded the naturally wake up time.

A survey was then filled out with questions on the clarity of dreams (see Self-Questionnaire). The wake-up time and the interval between wake-up time and last REM sleep was recorded. Questions about the level of clarity were included in the survey as well.

\section{Self-Questionnaire}

Date:

1. Did you wake up naturally or by an alarm?

Naturally wake up

By an alarm

2. From which stage of sleep did you wake up from?

REM Light Deep

3. If you did not wake up from REM, how long was it from the last REM?

4. When you wake up, can you recall your dreams? Yes No

5. On a scale of 5, what's the level of clarity of the dream?

Level 1 (The dream was not clear, and barely anything could be remembered)

Level 2 (The dream was kind of clear, some elements or fragments could be recalled)

Level 3 (The dream was clear, a vague plot can be recalled, instead of fragments.)

Level 4 (The dream was explicit, the plot can be fully recognized, as well as characters and places.)

Level 5 (The dream was vivid, the setting and the surroundings are very realistic, and the characters are also real.)

6. On a scale of 5, what's the level of connectivity of the dream to your life?

Level 1 [There is no connection between the dream and life]

Level 2 [A few elements/objects are similar to real life counterparts]
Level 3 [You can find objecrs/characters/identities from real life, but their relations were random and imaginary.]

Level 4 [The plot of the dream was similar or had connection with some real life events which follows certain patterns in real life]

Level 5 [The people/settings/plot of the dream clings to events happened in real life, like a redo of certain events]

7. If there a strong emotion behind the dream?

Yes No

8. If yes, please briefly explain that emotion.

9. Attached entry for any other important information (eg. physical exercise / caffeine or tea consumption / major events during the day/ the dream itself / etc.)

\subsection{Participant}

The investigation was conducted in the format of introspection. The only participant to be introspected was the researcher himself. The reasons behind the limited target of introspection were that the pandemic restrains chances of gathering people for offline experiments and sharing apparatus during the pandemic was not feasible.

\section{ANALYSIS}

There were totally seven nights of data collected, with detailed survey completed. The survey results were digitalized for further correlation analysis. The focus was the interval time between REM and waking up, and the level of clarity evaluated by the participant. On the survey, the participants chose from level 1 to level 5 sequentially as a scale of evaluation of the clarity of dream recalls (see Table 1.). The numbers were used to calculate the correlation between interval time and clarity of dream recall.

Table 1. The Interval Time and Dream Recall Clarity

\begin{tabular}{|l|l|l|}
\hline Date & $\begin{array}{l}\text { Interval Time } \\
(\mathrm{min})\end{array}$ & Level of Clarity \\
\hline 7.22 .2021 & 0 & 5 \\
\hline 7.23 .2021 & 5 & 5 \\
\hline 7.27 .2021 & 10 & 4 \\
\hline 7.28 .2021 & 220 & 2 \\
\hline 8.02 .2021 & 40 & 5 \\
\hline 8.05 .2021 & 110 & 0 \\
\hline 8.10 .2021 & 40 & 0 \\
\hline
\end{tabular}


The scatterplot of the data is shown below. There were seven nights' data in total. A trend line wass graphed with a function of $f(x)=-0.015 x+3.9102$. This trend line has a gradient of -0.015 , which illustrate a moderate negative correlation between the level of clarity of dreams and the interval time between last REM and the time waking up as the correlation coefficient. The $\mathrm{R}$ value is 0.52 (to 2 d.p.) with the P value as 0.11 (to 2 d.p.). Since the $\mathrm{P}$ value is greater than 0.05 , these data indicates that the data might occur at a relatively random chance. The $P$ value around 0.11 suggested a borderline negative correlation.

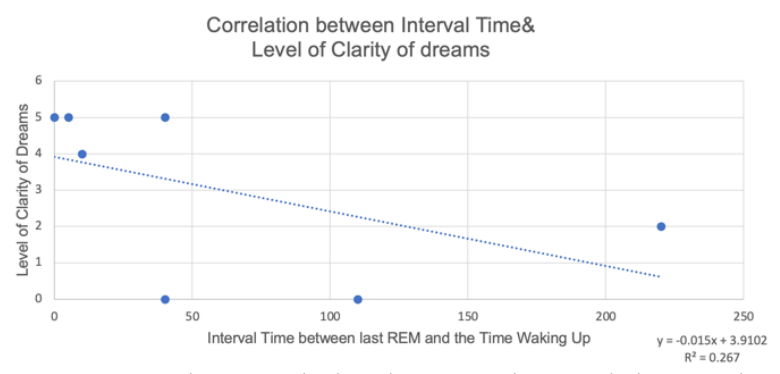

Figure 1 The correlation between interval time and level of clarity of dreams.

\section{CONCLUSION}

This investigation was conducted under COVID situations, where the public policies did not encourage investigation on a larger scale that include more participants. However, with the existing materials and opportunities, this investigation reveals a borderline negative relationship between the level of clarity of dream recalls and the interval time between last REM and the time waking up. In other words, the closer one woke up from REM sleep, the clearer they were to remember their dreams.

A previous study done on the individual difference in dream recall in 1996 found that 93 out of 100 people who were "dream reporters" reported themselves dreaming with contents while being waked up from REM stage of sleep [2]. The exceeding percentage of people reporting dream demonstrated that people tend to dream the most at REM stage and are likely to be able to recall them. Not only so, the "dream reporters" were able to give coarse recall on detailed content, objects and fragments in specifics [2]. Since all participants were waked up at their REM sleep, the interval of the time waking up and their REM sleep approaches to 0 . This explains that people might have the abilities to recall their dream clearly if they were waked up from their REM sleep or a time point close to that stage. The result in the research conducted by Helen Lewis helps explain and support this investigation to some degree. Therefore, the question came down to whether waking up at REM sleep enable people to recall their dreams most clearly, and what will the effect of longer interval time between waking up and REM sleep on the ability to recall dreams be.
In another research conducted on the malleability of dreams over time, the results recorded that $85 \%$ out of 26 people failed to recall suggested elements after 1 2 weeks interval from the first time they recalled dreams. The author explained that result in terms of stimulus which contributed to the formation of false memory which eventually falsified the recall of memory [3]. The interval time participants recall their dreams in BeaulieuPrévost's research was 1 2 week, which is much longer than the interval time in this investigation. However, their results did show a negative correlation of ability to recall dreams over time, similar to this investigation. Not only so, the explanation provided in Beaulieu-Prévost's research also gives an insight to reasons behind the loss of ability in recalling dreams found in this investigation. From Beaulieu-Prévost's understanding, many events between waking up and the REM sleep might have twisted the content of the dreams, which led to failures in recalling the dream clearly. For example, the sound of alarm or the noise from the kitchen during REM could make dreams harder to recall. However, more in-depth studies are needed in order to provide strong evidence to support this explanation.

While external stimulus potentially provides a possible explanation, strong emotions during the dreams, and before falling asleep, might be another influencing factor that led to this result. Study reveals that grief intensity has an indirect effect on dream recall [5]. Another research on empirical data of dream recall also suggests that major life events, mood before bed, and waking up during the night also might influence the quality and chance of dream recall [6].

Further improvements on this investigation could be on the amount of data and the variety of sample space. To be more specific, more trials could be collected, and different kinds of participants could be chosen instead of one person introspecting himself/herself. Increasing sample size would give us more confidence in our conclusion. Also, future investigations can focus on other influencing factors on dream recalls.

\section{REFERENCES}

[1] Stuff, H. (2020, October 26). Stages of sleep. Stages of Sleep | Michigan Medicine. Retrieved from https://www.uofmhealth.org/healthlibrary/hw48331.

[2] Lewis, H. B., Goodenough, D. R., Shapiro, A., \& Sleser, I. (1996). Individual differences in dream recall. American Psychological Association. Retrieved August 17, 2021, from https://psycnet.apa.org/search/display?id=4a6a97ad -aa18-3dab-af52-

10d57e504215\&recordId=9\&tab=PA\&page $=1 \&$ dis play $=25 \&$ sort $=$ PublicationYearMSSort + desc $\% 2 C$ AuthorSort+asc\&sr=1. 
[3] Beaulieu-Prévost, D., \& Zadra, A. (2015). When people remember dreams they never experienced: $A$ study of the malleability of dream recall over time. American Psychological Association. Retrieved from https://psycnet.apa.org/record/2015-05644001 ? doi $=1$.

[4] Novak, D. (2020). Muse S Meditation Headband. Muse S Meditation Headband - Immersive MultiSensor Meditation Device. Retrieved from https://www.gadgetgram.com/2020/09/28/muse-smeditation-headband-multi-sensor-meditationdevice/.

[5] Black, J., Belicki, K., \& Emberley-Ralph, J. (2019). Who dreams of the deceased? The roles of dream recall, grief intensity, attachment, and openness to experience. American Psychological Association. Retrieved from https://psycnet.apa.org/doiLanding?doi=10.1037\% 2Fdrm0000100.

[6] Schredl, M. (2007). Dream Recall: Models and Empirical Data. American Psychological Association. Retrieved from https://psycnet.apa.org/record/2007-09896-004.

[7] Siegel, J., \& Rechtschaffen, A. (2000). Sleep and Dreaming. Rechtschaffen, a. Retrieved August 17, 2021, from https://www.semel.ucla.edu/sites/default/files/sleep /publications/sleep_dreams.htm.

[8] Yu, C. K. C. (2020). We dream about typical themes in both REM and non-REM sleep. American Psychological Association. Retrieved from https://psycnet.apa.org/doiLanding?doi=10.1037\% 2Fdrm0000154. 